УДК 548.945

\title{
Modeling the dc Current Flow in Granular Superconductor Wires
}

\author{
Mikhail V. Belodedov* \\ Bauman Moscow State Technical University \\ 2 baumanskaya, 5/1, Moscow, 105005 \\ Russia \\ Levan P. Ichkitize ${ }^{\dagger}$ \\ National Research University of Electronic Technology \\ Shokina, 1, Zelenograd, Moscow, 124498
}

Russia

Received 03.11.2016, received in revised form 29.12.2016, accepted 06.02.2017

\begin{abstract}
Granular superconductor considered as a multiple Josephson medium, for modeling the electrodynamics of which the averaged material equation has being proposed. Evaluations of the critical parameters of the medium (the magnetic field penetration depth and the first critical field) are been obtained. The simulation of the current flowing through the granular superconductor wires was conducted. The presence of the skin effect in such wires at lowest frequencies down to DC is shown. The recommendations are made to use the stranded wires for the transmission of high currents.
\end{abstract}

Keywords: ceramic superconductor, critical current, size effect, josephson junction.

DOI: 10.17516/1997-1397-2017-10-2-239-243.

Application of high-temperature superconducting ceramics (HTSC) is constrained by a lack of adequate models of HTSC, as a multiple Josephson medium. It is generally accepted [1] to consider this medium as a stochastic set of superconducting grains with Josephson junctions at their contact points. Averaging the of Ginzburg-Landau equation[2] over a small volume containing a large number of grains, one can develop the material equation [3] that relates the averaged current density vector $\mathbf{j}$ in the medium and the magnetic-field vector potentia $\mathbf{F}$ :

$$
\mathbf{j}=-\frac{\mathbf{F}}{F} I_{c} \rho a \frac{\pi}{8}\left(\frac{2 \pi}{\Phi_{0}} F a\right) \exp \left[-\frac{\pi}{16}\left(\frac{2 \pi}{\Phi_{0}} F a\right)^{2}\right]-\frac{\rho}{R c} \frac{\pi a^{2}}{8} \frac{d \mathbf{F}}{d t}-\frac{C \rho}{c} \frac{\pi a^{2}}{8} \frac{d^{2} \mathbf{F}}{d t^{2}},
$$

where $\Phi_{0}=h c / 2 e=2.07 \cdots \times 10^{-7} G \times \mathrm{cm}^{2}$ is the magnetic flux quantum, $\rho$ is the concentration of Josephson junctions in a medium, $I_{c}, R$ and $C$ are the averaged critical current, active resistance and capacity of a single Josephson junction, and $a$ is the averag size of grains forming the junction.

The material equation is derived under the assumption of the Maxwell grain size distribution. However, as was shown by simulation of processes occuring in a multiple Josephson medium, their character remains invarible at the other grain size distributions.

Using Eq. (1), we simulated penetration of magnetic field into bulk HTS samples. It was established that the magnetic field penetrates into the sample to a depth of

$$
\lambda_{M}=\sqrt{\frac{\Phi_{0}}{2 \pi} \frac{2 c}{\mu I_{c} \rho \pi^{2} a^{2}}},
$$

\footnotetext{
*m.belodedov@mail.ru

$\dagger$ leo852@inbox.ru

(c) Siberian Federal University. All rights reserved
} 
where $\mu$ is the magnetic permeability of the medium caused only by the Meissner currents of individual grains.

In addition, it was shown that in bulk HTSC the magnetic vortices with size $\lambda_{M}$ containing single magnetic flux quanta (the so-called hypervortices [1]) are the most energetically favorable [1]. According to the data of numerical simulation, the maximum external magnetic field at which there no hypervortices in a medium is

$$
B_{1}=2.25676 \ldots \times \frac{\Phi_{0}}{2 \pi a \lambda_{M}} .
$$

The maximum current flowing through a HTS wire is of great practical interest. The proposed material equation allows us to obtain some results in this direction.

Let us consider an infinitely long cylindrical sample with radius $R$ made of the above-described granular medium. Introducing polar coordinates with the $z$ axis directed along the sample axis, we can reasonably assume that the variable $\mathbf{F}$ has the only component directed along the $z$ axis and depending only on $r: \mathbf{F}(\mathbf{r})=F(r)$. In this case, simulation of the stationary current distribution in the sample is reduced to solving the equation

$$
\frac{d}{d r}\left(\frac{1}{r} \frac{d}{d r}(r F(r))\right)=\frac{\Phi_{0}}{2 \pi} \frac{1}{a \lambda_{M}^{2}} M(F),
$$

where the material function

$$
M(F)=\left(\frac{2 \pi}{\Phi_{0}} F a\right) \exp \left[-\frac{\pi}{16}\left(\frac{2 \pi}{\Phi_{0}} F a\right)^{2}\right],
$$

was introdused. When the vortices are missing, the equation is added with the boundary condition $\lim _{x \rightarrow 0}(r F)=0$.

Since solving the second-order differential equation requires two boundary conditions, we should impose the additional condition $F(R)=F_{R}$, which will allow us to find the equation solution $F(r)$. Then, the current distribution in the sample will be specified by the relation

$$
j=-\frac{\pi I_{c} \rho a}{8} M(F),
$$

and the total current through the sample will be expressed as

$$
I=2 \pi \int_{0}^{R} j(r) r d r
$$

To determine the maximum possible current through the sample, it is sufficient to find the maximum total current at all possible $F_{R}$ values:

$$
I_{\max }=2 \pi \max _{F_{R}}\left(\int_{0}^{R} j(r) r d r\right) .
$$

Thus, using the numerical simulation, one can easily obtain the dependence of the maximum current in a granular superconductor wire on its diameter $D=2 R$. The results of numerical simulation of differential equation (2) for the samples with different diameters (in units of $\lambda_{M}$ ) are shown in Fig. 1. The current flowing through the sample is concentrated mainly in a thin surface layer with thickness of about $\lambda_{M}$; thus, the dc skin effect is observed. As a result, the dependence of the total current in the sample on its diameter at $D>\lambda_{M}$ should be almost linear, which is confirmed by the numerical experiments (Fig. 2). 
Similarly, we can analyze the stationary current distribution in a thin $\left(b \ll \lambda_{M}\right)$ granular superconductor strip. In this case, the vector $\mathbf{F}$ should be assumed to have the only component directed along the strip and depending only on coordinate $y$ perpendicular to the strip direction and obeying the equation

$$
\frac{d^{2}}{d y^{2}} F(y)=\frac{\Phi_{0}}{2 \pi} \frac{1}{a \lambda_{M}^{2}} M(F),
$$

and the current distribution in the strip will still be determined by expression (3):

$$
j=-\frac{\pi I_{c} \rho a}{8} M(F)
$$

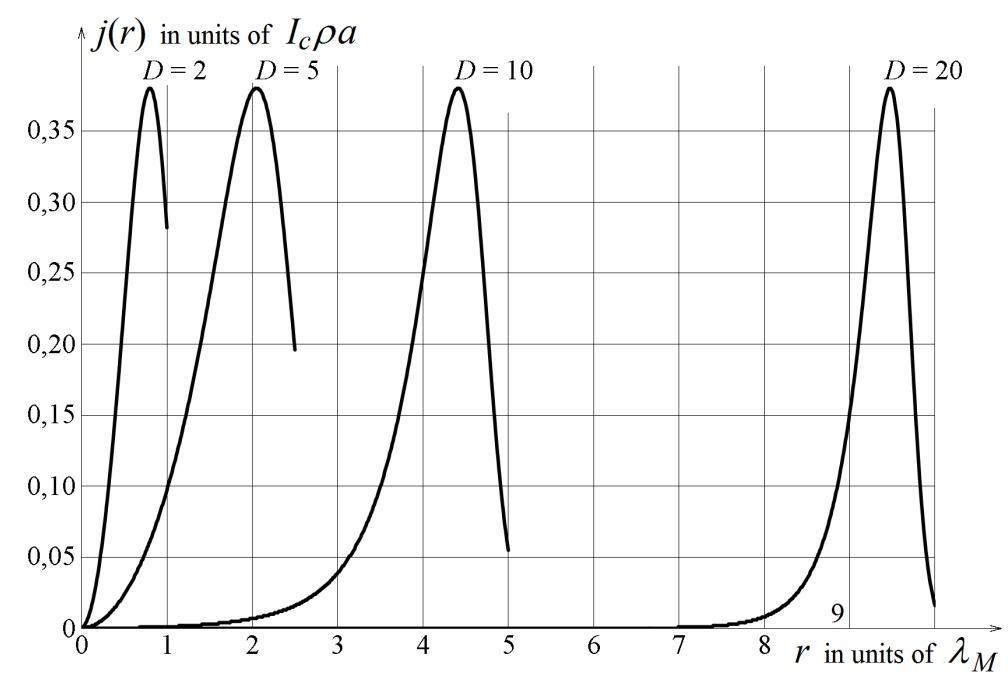

Fig. 1. Stationary current density distribution in cylindrical wires of granular superconductors with different diameter $D$

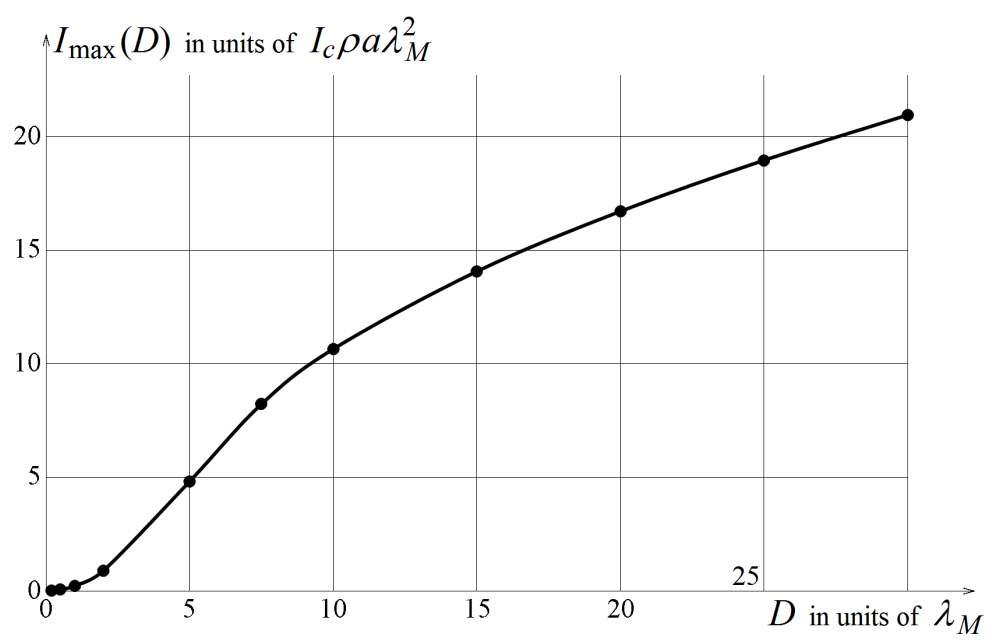

Fig. 2. Dependence of the maximum current in a cylindrical granular superconductor wire on its diameter $D$ 
The totall current will be expressed as

$$
I=b \int_{-D / 2}^{D / 2} j(y) d y .
$$

Solving Eq. (4) with the boundary conditions

$$
F(D / 2)=F(-D / 2)=F_{D / 2},
$$

one can select the $F_{D / 2}$ value at which the total current through the strip will acquire the maximum value:

$$
I_{\max }=\max _{F_{D / 2}}\left(b \int_{-D / 2}^{D / 2} j(y) d y\right) .
$$

Numerical simulation of differential equation (4) with the requirement for the maximum total current yields the current distributions in strip samples of different widths shown in Fig. 3. As in cylindrical wires, the current is concentrated in a narrow boundary region with a width of about $\lambda_{M}$, which confirms once again the presence of the dc skin effect.

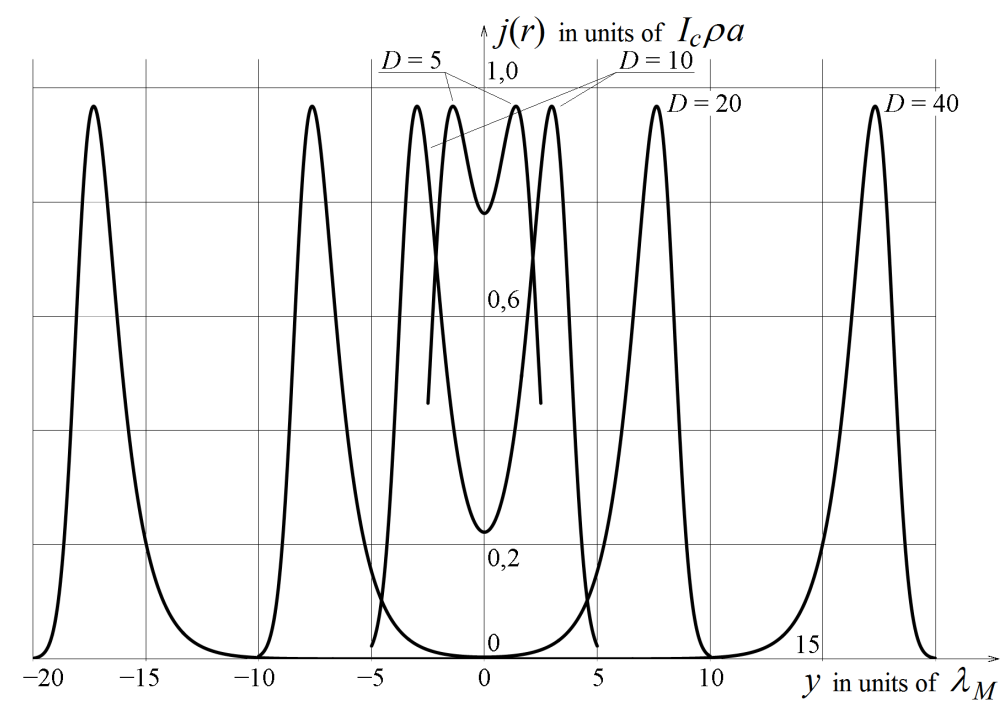

Fig. 3. Stationary current density distribution in strip wires of granular superconductors with a different widths $D$

The presence of the skin effect in strip wires should lead to the situation when at large (as compared to $\lambda_{M}$ ) strip widths the maximum current through the wire tends to a constant value. This is confirmed by the data plotted in Fig. 4.

The results obtained allow us to conclude that the skin effect that manifests itself at low frequencies up to the dc current is of great importance for the HTSC consisting of granular superconducting media. Therefore, to achieve the maximum possible currents through HTSC wires (both bulk and strip), it is reasonable to fabricate them in the litzendraht form consisting of a large number of unbound conductors with a thickness of no more than several $\lambda_{M}$.

This work was supported by the Ministry of Education and Science of the Russian Federation, agreement No. 14.581.21.0014 (Project ID RFMEFI58115X0014). 


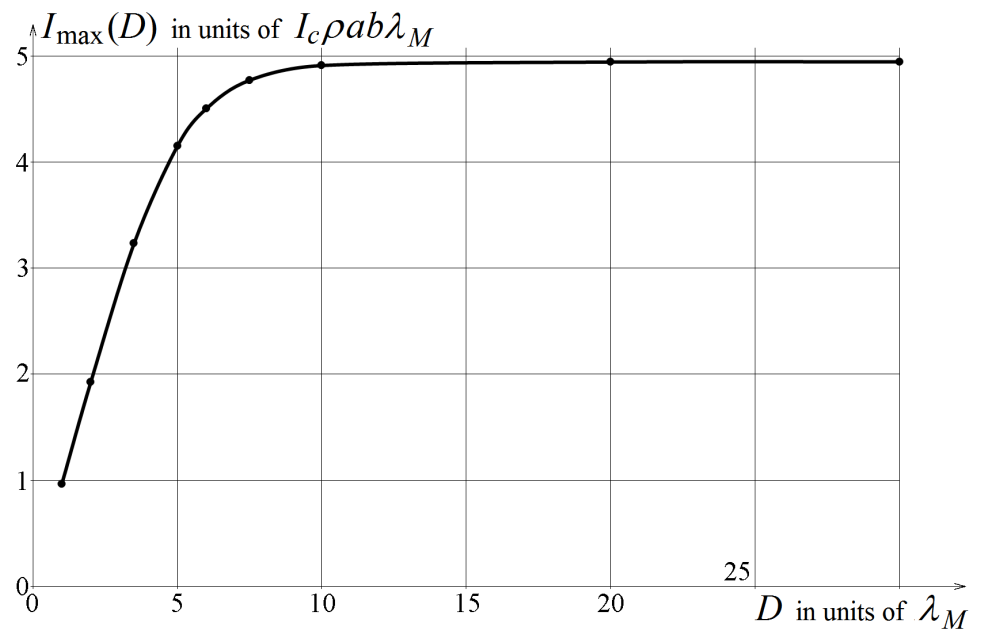

Fig. 4. Dependence of the maximum current in a strip wire of a granular superconductor on its width $D$

\section{References}

[1] E.B.Sonin, The "Josephson medium" theory in HTSC: vortices and critical magnetic fields, JETP Lett., 47(1988), no. 8, 415-418.

[2] V.V.Schmid, Introduction to Physics of Superconductors, 2000, 2nd edition, Rev. and extra, MCNMO.

[3] M.V.Belodedov, L.P.Ichkitidze, Material equation for granular superconductors, Izhenernyi zhurnal: nauka i innovatsii, 22(2013), no. 10 (in Russian).

\section{Моделирование протекания тока через провода из гранулярного сверхпроводника}

Михаил В. Белодедов

МГТУ им. Н.Э. Баумана

2-я Бауманская, 5/1, Москва, 105005

Россия

Леван П. Ичкитидзе

Национальный исследовательский университет МИЭТ Шокина, 1, Зеленоград, Москва, 124498

Россия

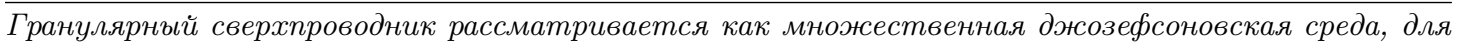
моделирования электродинамики которой предлагается усреднённое материальное уравнение. Получены оценки критических параметров среды (глубина проникновения магнитного поля и первое критическое поле). Проведено моделирование протекания тока через провода из гранулярного сверхпроводника. Показано наличие скин-эффекта в таких проводах на низких частотах вплоть до постоянного тока. Даётся рекомендация использовать многожильные провода для передачи больших токов.

Ключевые слова: керамические сверхпроводники, критический ток, размерный эффект, джозефсоновский переход. 